\title{
Fixed Points and Stability Analysis in the Motion of Human Heart Valve Leaflet
}

\author{
Emagbetere, Eyere ${ }^{1,}$, Salau, A.O. Tajudeen ${ }^{2}$ and Oluwole, Oluleke ${ }^{2}$ \\ Mechanical Engineering Department, Federal University of Petroleum Resources, Effurun, Delta \\ State, Nigeria \\ Mechanical Engineering Department, University of Ibadan, Ibadan, Nigeria \\ emagbetere.eyere@fupre.edu
}

Keywords: Heart valve model, phase portraits, numerical simulation, transcritical bifurcation

\begin{abstract}
This work was set out to gain further insight into the kinetics of the human heart valve leaflet. The Korakianitis and Shi lumped parameter model was adopted for this study. The fixed points were determined, and then, their stability properties were assessed by evaluating eigenvalues of the Jacobian matrices. Normal physiological parameters for the valve model were simulated; based on which, a local bifurcation diagram was generated. Phase portraits were plotted from simulated responses, and were used to observe the qualitative properties of the valve leaflet motion. The evaluated fixed points were found to be dependent on pressure and flow effects, and independent on friction or damping effect. Observed switching of stability between the two fixed points indicated that the leaflet motion undergoes transcritical bifurcation. Of the two fixed points, one is always either a stable spiral or generative node while the other is a saddle. Numerical simulations were carried out to verify the analytical solutions.
\end{abstract}

\section{Subject Classification Numbers: (MSC2010) 74L15, 70E17, 37N15}

\section{Introduction}

The human heart valves have leaflets which open and close passively to control unidirectional blood flow. Although normal leaflets motion is expected for both native and prosthetic valves, loss in mobility has been observed in certain valve types [1-4]. Whether severe or mild, any form of abnormal leaflet motion is said to render the valve structurally dysfunctional [3]. While it is important to characterise the clinical incidence and risk of abnormal leaflets motion, there is also the need for further investigation into the dynamics of valve leaflets motion.

Experimental assessment of valve leaflets' motion started out with the attachment of film probes to animal models and obtaining velocity profile using anemometer [5, 6]. This was followed by the attachment of markers to animal models and tracking with x-rays to obtain velocity profiles of the valve leaflets $[7 ; 8]$. These techniques were applied mainly on animal models and cannot sufficiently study human heart valve dynamics. Additionally, they are not feasible for clinical studies. The introduction of laser scanners for quantifying valve motion [9] was a good replacement for attached markers. Via laser profiling, the deformations of the valve leaflets under different flow conditions were successfully studied $[10,11]$. Over the years, clinical scanners had gained improvements and were used to assess valve motion both for clinical studies and diagnostic purposes. There are current sophisticated imaging techniques such as the Computed Tomography (CT). Very recently, leaflet motion of certain prosthetics heart valves was widely studied using CT scanners [2, $3,12,13]$. It is noteworthy that imaging techniques cannot be used to assess the kinetics (forces and effect on motion) of the valve leaflets. Furthermore, assessment of long term variation in dynamical variables of the heart valve motion cannot be achieved using current imaging techniques.

Combination of imaging techniques and mathematical modelling has been extensively used to achieve impressive success in the study of heart valve dynamics. There are a number of review articles that reported some of these computational models; their development, applications and limitations [14-16]. There are recent highly sophisticated 3D fluid structure interaction models for heart valves 
dynamics [17-23]. These models were used to simulate vascular stresses, leaflets deformations, and hemodynamics of the heart valves. Some basic physics such as the kinetics of global leaflet motion may be obscured in such simulations. To capture such physics, one may resort to a simple zerodimensional model. A number of these zero-dimensional models can be seen in the review by Shi et al. [24]. These zero-dimensional models are usually coupled with other models to assess global cardiac responses [25-27]. There is death of information on the nature of the valve leaflet motion in a state space and the possible qualitative dynamical changes that can occur.

The aim of this work is to investigate the equilibrium position of the valve leaflets, its, stability properties, and local bifurcation, using a valid heart valve model. We arranged the other section of this paper as follows; the second section started out with a brief description of the adopted model followed by an evaluation of the fixed points and stability analysis. In the third section, numerical results in form of phase portraits and a local bifurcation diagram for a range of parameter set were presented. The last section is a discussion and concluding remark based on the results.

\section{Methodology}

\subsection{The Model}

The Korakianitis and Shi (KS) lumped parameter model was adopted for this study. The model consists of a heart valve model which is embedded in the overall model of the heart chambers, and the pulmonary and systemic loops. The heart functions as a pump having two parts. One part (right heart) is responsible for pumping blood through the lungs (to be mixed with oxygen) and back to the heart. The other part (left heart) pumps the oxygenated blood through the entire body. Each part has an upper chamber (atrium) and a lower chamber (ventricle). Both chambers are separated by the Atrio-Ventricular (AV) valves. The ventricle (lower chamber) is separated from the sinus which leads out of the heart by the semilunar valves. The different parts of the circulatory system which the blood goes through before returning to the heart are: the sinus, arteries and veins. Further details can be found in the literature [29, 30]. The governing equations for the different parts of the circulatory system and their solutions are presented in this section. The parameters for the model are shown in Table 1.

\subsubsection{Equation for the Heart Valve}

The KS heart valve model captures most of the factors that influence the motion of heart valve leaflet, and has successfully been used to simulate the valve motion within a heart cycle [28]. The factors that influence the motion of the heart valve leaflet are blood-flow effects of: pressure gradient $\left(F_{p}\right)$, friction effect due to tissue resistance $\left(F_{f}\right)$, effect of blood motion $\left(F_{b}\right)$, action of downstream blood vortex $\left(F_{v}\right)$ and the leaflet shear stresses $\left(F_{\tau}\right)$. These effects can be envisioned in Fig. 1 .

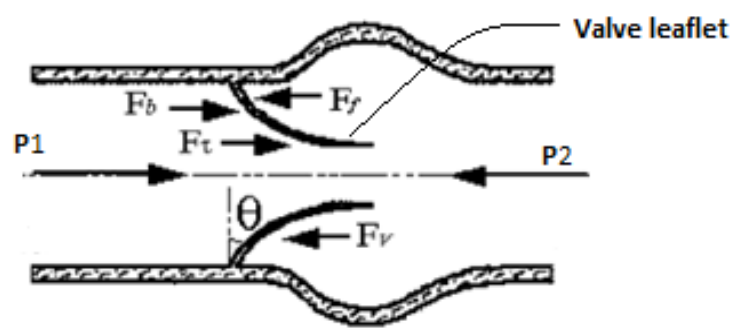

Figure 1. Two dimensional description of angular displacement of valve leaflet Source: [28]

Inertial torque is proportional to the sum of all factors influencing the leaflet motion. This is based on the principle of dynamic equilibrium or conservation of momentum. Hence, Equation 2.1 is written out

$$
I \ddot{\theta}=F_{p}-F_{f}+F_{b}-F_{v}+F_{\tau}
$$


$I \ddot{\theta}$ is the inertia force, where $\ddot{\theta}$ is the angular acceleration of the valve. KS modelled the different effects base on the following considerations: $F_{p}$ is proportional to the normal pressure effect on the leaflet surface; $F_{f}$ is proportional to the angular velocity of the valve leaflet; $F_{b}$ is proportional to blood flow rate $(Q)$ normal to the leaflet surface; $F_{v}$ is proportional to a component blood flow rate that is maximum at a 45 degree position of the valve leaflet; $F_{\tau}$ is negligible.

The equation describing the position of the valve leaflets was then derived as shown in equation 2.2

$$
\ddot{\theta}=\left(P_{1}-P_{2}\right) K_{p} \cos (\theta)-K_{f} \dot{\theta}+K_{b} Q \cos (\theta)-K_{v} Q \sin (\theta)
$$

\subsubsection{Equations for the Heart Chambers}

$$
\begin{aligned}
& \frac{d V}{d t}=Q_{\text {in }}-Q_{\text {out }} \\
& V=\left(Q_{\text {in }}-Q_{\text {out }}\right) \Delta t+V^{\prime} \\
& P_{t}=P_{\text {min }}+E\left(V-V_{\text {min }}\right)
\end{aligned}
$$

where $\mathrm{E}$ is given as $\mathrm{E}_{\mathrm{a}}$ for atrium and $\mathrm{Ev}$ for ventricle calculated with the equations below.

$$
\begin{aligned}
& E_{V}=E_{v, d}+\frac{E_{v, s}-E_{v, d}}{2} e_{v}(t) \\
& e_{v}(t)=\left\{\begin{array}{cc}
\operatorname{Cos}\left(\frac{t}{T_{s 1}} \pi\right), & 0 \leq t<T_{s 1} \\
\cos \left(\frac{t+T_{s 2}-T_{s 1}}{T_{s 1}} \pi\right), & T_{s 1} \leq t<T_{s 2} \\
0, & T_{s 2} \leq t<T
\end{array}\right. \\
& E_{a}=E_{a, \text { min }}+\frac{E_{a, \max }-E_{a, \min }}{2} e_{a}(t) \\
& e_{a}(t)=\left\{\begin{array}{cc}
0, & 0 \leq t<T_{p b} \\
1-\cos \left(\frac{t+T_{p b}}{T_{p w}} 2 \pi\right), & T_{p b} \leq t<T_{p b}+T_{p w} \\
0, & T_{p b}+T_{p w} \leq t<T
\end{array}\right.
\end{aligned}
$$

\subsubsection{Equations for the Sinus}

The instantaneous pressure in the aortic sinus (Pas) was modelled using equation 2.7a while equation 2.8 a was used to describe the flow through the aortic sinus.

$$
\frac{d P_{a s}}{d t}=\frac{Q_{a v}-Q_{a s}}{C_{a s}}
$$

Solved by direct integration to yield

$$
\begin{aligned}
& P_{a s}=\left\{\frac{Q_{a v}-Q_{a s}}{C_{a s}}\right\} \Delta t+P_{a s}^{\prime} \\
& \frac{d Q_{a s}}{d t}=\frac{P_{a s}-P_{a t}-R_{a s} Q_{a s}}{L_{a s}}
\end{aligned}
$$




$$
Q_{s a s}=\frac{\left(P_{a s}-P_{a t}\right)}{R_{a s}}+\left(Q_{a s}^{\prime}-\frac{\left(P_{a s}-P_{a t}\right)}{R_{a s}}\right) \cdot \exp \left(-\frac{\mathrm{R}_{\mathrm{as}}}{L_{a s}} \cdot \Delta t\right)
$$

\subsubsection{Equations of the Arteries}

Equations 2.9a and 2.10a govern the pressure and flow in the arteries respectively.

$$
\frac{d P_{a t}}{d t}=\frac{Q_{a s}-Q_{a t}}{C_{a t}}
$$

Solved by direct integration

$$
\begin{aligned}
P_{a t} & =\left(\frac{Q_{a s}-Q_{a t}}{C_{a t}}\right) \Delta t+P_{a t}^{\prime} \\
\frac{d Q_{a t}}{d t} & =\frac{P_{a s}-P_{v n}-\left(R_{a t}+R_{a}+R_{c p}\right) Q_{a t}}{L_{a t}}
\end{aligned}
$$

Integrated by separating the variables.

$$
Q_{s a t}=\frac{\left(P_{a t}-P_{v n}\right)}{\left(R_{a t}+R_{a}+R_{c p}\right)}+\left(Q_{a t}^{\prime}-\frac{\left(P_{a t}-P_{v n}\right)}{\left(R_{a t}+R_{a}+R_{c p}\right)}\right) \cdot \exp \left(-\frac{R_{a t}+R_{a}+R_{c p}}{L_{a t}} \cdot \Delta t\right)
$$

\subsubsection{Equations of the Veins}

Equations 2.11 and 2.12 govern the pressure and flow in the veins respectively.

$$
\begin{aligned}
& \frac{d P_{v n}}{d t}=\frac{Q_{a t}-Q_{v n}}{C_{v n}} \\
& P_{v n}=\left(\frac{Q_{a t}-Q_{v n}}{C_{v n}}\right) \Delta t+P_{v n}^{\prime} \\
& \frac{d Q_{v n}}{d t}=\frac{P_{v n}-P_{a}}{R_{v n}} \\
& Q_{v n}=\left(\frac{P_{v n}-P_{a}}{R_{v n}}\right) \Delta t+Q_{v n}^{\prime}
\end{aligned}
$$

\begin{tabular}{|c|c|c|c|c|c|}
\hline Parameters & & & & & \\
\hline $\begin{array}{l}\text { Valve } \\
\text { Model }\end{array}$ & $\begin{array}{l}\mathrm{Kp} \\
(\mathrm{rad} / \mathrm{s} 2 \mathrm{mmHg})\end{array}$ & $\mathrm{Kf}(1 / \mathrm{s})$ & $\mathrm{Kb} \mathrm{rad} /(\mathrm{sm})$ & \begin{tabular}{|l|}
$\mathrm{Kv}$ \\
$(\mathrm{rad} / \mathrm{sm})$
\end{tabular} & \\
\hline Mitral & 5500 & 50 & 2 & 3.5 & \\
\hline Tricuspid & 5500 & 50 & 2 & 3.5 & \\
\hline Aortic & 5500 & 50 & 2 & 7 & \\
\hline Pulmonary & 5500 & 50 & 2 & 3.5 & \\
\hline $\begin{array}{l}\text { Heart } \\
\text { chamber }\end{array}$ & $\begin{array}{l}\text { Pmin,a } \\
(\mathrm{mmHg})\end{array}$ & Vmin,a (ml) & $\begin{array}{l}\text { Pmin, } \mathrm{V} \\
(\mathrm{mmHg})\end{array}$ & $\begin{array}{l}\text { Vmin, } \mathrm{V} \\
(\mathrm{ml})\end{array}$ & \\
\hline Systemic & 1 & 5 & 1 & 5 & \\
\hline Pulmonary & 1 & 4 & 1 & 10 & \\
\hline Elastance & $\begin{array}{l}\text { Ea,min } \\
(\mathrm{mmHg} / \mathrm{ml})\end{array}$ & \begin{tabular}{|l|}
$\begin{array}{l}\mathrm{Ea}, \mathrm{max} \\
(\mathrm{mmHg} / \mathrm{ml})\end{array}$ \\
\end{tabular} & $\begin{array}{l}\text { Ev,d } \\
(\mathrm{mmHg} / \mathrm{ml})\end{array}$ & \begin{tabular}{|l}
$\begin{array}{l}\mathrm{Ev}, \mathrm{s} \\
(\mathrm{mmHg} / \mathrm{ml})\end{array}$ \\
\end{tabular} & \\
\hline Systemic & 0.15 & 0.25 & 2.5 & 0.1 & \\
\hline Pulmonary & 0.15 & 0.25 & 1.15 & 0.1 & \\
\hline Timing & $\mathrm{Tpb}(\mathrm{s})$ & Tpw (s) & Ts1 (s) & Ts2 (s) & \\
\hline & 0.92 & 0.09 & 0.3 & 0.6 & \\
\hline
\end{tabular}

Table 1. Model Parameters 


\begin{tabular}{|c|c|c|c|c|c|}
\hline $\begin{array}{l}\text { Blood } \\
\text { vessels }\end{array}$ & $\begin{array}{l}\text { Cas } \\
(\mathrm{ml} / \mathrm{mmHg})\end{array}$ & $\begin{array}{l}\text { Cat } \\
(\mathrm{ml} / \mathrm{mmHg})\end{array}$ & $\begin{array}{l}\text { Cvn } \\
(\mathrm{ml} / \mathrm{mmHg})\end{array}$ & $\begin{array}{l}\text { Ccp } \\
(\mathrm{ml} / \mathrm{mmHg})\end{array}$ & \\
\hline Systemic & 0.08 & 1.6 & 20.5 & 1.5 & \\
\hline Pulmonary & 0.18 & 3.8 & 20.5 & 1.5 & \\
\hline & $\begin{array}{l}\text { Ras (mmHg- } \\
\mathrm{s} / \mathrm{ml})\end{array}$ & $\begin{array}{l}\text { Rat (mmHg- } \\
\mathrm{s} / \mathrm{ml})\end{array}$ & $\begin{array}{l}\text { Rvn } \\
(\mathrm{mmHg}- \\
\mathrm{s} / \mathrm{ml})\end{array}$ & $\begin{array}{l}\mathrm{Rcp} \\
(\mathrm{mmHg}- \\
\mathrm{s} / \mathrm{ml})\end{array}$ & $\begin{array}{l}\text { Rar } \\
(\mathrm{mmHg}- \\
\mathrm{s} / \mathrm{ml})\end{array}$ \\
\hline Systemic & 0.03 & 0.05 & 0.075 & 0.52 & 0.5 \\
\hline Pulmonary & 0.02 & 0.01 & 0.006 & 0.25 & 0.05 \\
\hline & $\begin{array}{l}\text { Las (mmHg- } \\
\text { s } 2 / \mathrm{ml})\end{array}$ & $\begin{array}{l}\text { Lar (mmHg- } \\
\text { s2/ml) }\end{array}$ & & & \\
\hline Systemic & 0.000062 & 0.0017 & & & \\
\hline Pulmonary & 0.000052 & 0.0017 & & & \\
\hline
\end{tabular}

\subsection{Evaluation of Fixed Points}

The Fixed points can also be called the stationary points or equilibrium points. It is an intending rest position of a displaced body or part of a body, as influenced by the certain forcing parameters.

To investigate the fixed points point, Equation 2.2 was reduced as to:

$$
\ddot{\theta}=A \cos (\theta)-B \sin (\theta)-C \dot{\theta}
$$

where, $A=\left\{\left(P_{1}-P_{2}\right) K_{p}+K_{b} Q\right\}$, is the instantaneous effect of pressure and blood flow. $B=$ $\left\{K_{v} Q \sin \right\}$ is the instantaneous effect of underlying vortex or any other effect that may be underneath the leaflet such as clothed blood, and $C=K_{f}$ Is the effect due to damping (tissue friction) of the valve.

If we make; $\theta_{1}=\theta$ and $\theta_{2}=\dot{\theta}$, then the system (Equation 2.13) becomes:

$$
\begin{aligned}
& \dot{\theta}_{1}=\theta_{2} \\
& \dot{\theta}_{2}=A \cos \left(\theta_{1}\right)-B \sin \left(\theta_{1}\right)-C\left(\theta_{2}\right)
\end{aligned}
$$

As discussed in literature $[32 ; 33 ; 34]$, the fixed points $\left(\theta^{*}\right)$ satisfy $\dot{\theta}_{1}=\dot{\theta}_{2}=0$ (where $\dot{\theta}_{1}$ and $\dot{\theta}_{2}$ are the velocity and acceleration respectively). Therefore,

$$
\begin{aligned}
& \theta_{2}^{*}=0 \\
& \frac{A}{B}=\tan \left(\theta_{1}^{*}\right)
\end{aligned}
$$

Thus

$$
\theta_{1}^{*}=\tan ^{\prime}\left(\frac{A}{B}\right)
$$

Considering that $\tan (\theta)$ has the same magnitude and sense on only two quadrants, It can be deduced from Equation 2.16 that the leaflet motion has two fixed points. The fixed points are given as $\left(\theta_{1}^{*}, 0\right)$ and $\left(\pi+\theta_{1}^{*}, 0\right)$, and lie between $-\frac{\pi}{2}$ and $\frac{\pi}{2}$.

\subsection{Linear Stability Analysis}

The stability properties of the fixed points were assessed by evaluating the eigenvalues of the Jacobian matrices. 


\subsubsection{Jacobian Matrix}

The Jacobian matrix $(\mathrm{J})$ is given by the equation below:

$$
J=\left(\begin{array}{ll}
\frac{\partial \dot{\theta}_{1}}{\partial \theta_{1}} & \frac{\partial \dot{\theta}_{1}}{\partial \theta_{2}} \\
\frac{\partial \dot{\theta}_{2}}{\partial \theta_{1}} & \frac{\partial \dot{\theta}_{2}}{\partial \theta_{2}}
\end{array}\right)_{\left(\theta_{1}{ }^{*}, \theta_{2}{ }^{*}\right)}
$$

From equation $2.14 \mathrm{a}$ and $2.14 \mathrm{~b}$, we have:

$$
\begin{aligned}
& \frac{\partial \dot{\theta}_{1}}{\partial \theta_{1}}=0 \\
& \frac{\partial \dot{\theta}_{1}}{\partial \theta_{2}}=1 \\
& \frac{\partial \dot{\theta}_{2}}{\partial \theta_{1}}=\frac{\partial\{A \cos (\theta 1)-B \sin (\theta 1)-C(\theta 2)\}}{\partial \theta_{1}}=-A \sin \left(\theta_{1}\right)-B \cos \left(\theta_{1}\right) \\
& \frac{d \dot{\theta}_{2}}{d \theta_{2}}=\frac{\partial\{A \cos (\theta 1)-B \sin (\theta 1)-C(\theta 2)\}}{\partial \theta_{2}}=-C
\end{aligned}
$$

One can deduce from equation 2.16 that

$$
\begin{aligned}
& \sin \left(\theta_{1}^{*}\right)=\frac{+A}{\sqrt{A^{2}+B^{2}}} \\
& \sin \left(\pi+\theta_{1}^{*}\right)=\frac{-A}{\sqrt{A^{2}+B^{2}}} \\
& \cos \left(\theta_{1}\right)=\cos \left(\theta_{1}^{*}\right)=\frac{+B}{\sqrt{A^{2}+B^{2}}} \\
& \cos \left(\pi+\theta_{1}^{*}\right)=\frac{-B}{\sqrt{A^{2}+B^{2}}}
\end{aligned}
$$

Putting together equations 2.17, 2.18a, 2.18b, 2.18c, 2.18d, 2.19a, 2.19b, 2.19c and 2.19d; the obtained Jacobian matrices $(\mathrm{J})$ is given as:

$$
\begin{aligned}
& J_{\theta_{1}^{*}}=\left[\begin{array}{cc}
0 & 1 \\
-\sqrt{A^{2}+B^{2}} & -C
\end{array}\right] \\
& J_{\left(\theta_{1}^{*}+\pi\right)}=\left[\begin{array}{cc}
0 & 1 \\
+\sqrt{A^{2}+B^{2}} & -C
\end{array}\right]
\end{aligned}
$$

\subsubsection{Eigenvalues}

Let

$$
D=\sqrt{A^{2}+B^{2}}
$$

From the Jacobian matrices (Equations 2.20a and 2.20b) the eigenvalues $(\lambda)$ is thus determined:

$$
\begin{aligned}
& \lambda_{\theta}^{2}+C \lambda_{\theta}+D=0 \\
& \lambda_{(\theta+\pi)}^{2}+C \lambda_{(\theta+\pi)}-D=0
\end{aligned}
$$

The Eigenvalues of the two fixed points is defined by Equations 2.22a and 2.22b: 


$$
\begin{aligned}
& \lambda_{\theta}=\frac{1}{2}\left(-C \pm \sqrt{C^{2}-4 D}\right) \\
& \lambda_{(\theta+\pi)}=\frac{1}{2}\left(-C \pm \sqrt{C^{2}+4 D}\right)
\end{aligned}
$$

Since the roots (Equations 2.23a and 2.23b) are not pure imaginary numbers, theorem exists to show that the local motion of the linearized system is qualitatively similar to the original nonlinear system [34]. Consequently, no further analysis is required to establish the stability of the nonlinear system.

\subsection{Numerical Simulation}

Numerical Simulations were carried out to obtain parameters for the valve model, and to generate phase portraits and bifurcation plots.

\subsubsection{Simulation of Physiological Parameters for the Human Heart Valve}

Since the values of A and B are dependent on changing pressure and flow over a heart cycle, their values for a heart cycle were first simulated using the following quasi-code for the cardiovascular system.

$\{$ Start

* Initialize all variables (System parameter and initial conditions)

* Do while no. of times < desired number of heart cycles

$\circ$ Do while $0<\Delta t<T$ ( $T$ is the heart period)

- $\quad$ Simulate blood pressure and flow in systemic and pulmonary sinus (Equations $2.7 b$ and $2.8 b)$

- $\quad$ Simulate blood pressure and flow in systemic and pulmonary artery (Equations $2.9 b$ and $2.10 b$ )

- $\quad$ Simulate blood pressure and flow in systemic and pulmonary veins (Equations $2.11 b$ and $2,12 b$ )

- $\quad$ Simulate blood pressure and flow though the systemic and pulmonary atria (Equations 2.3a, 2.3b, 2.4, 2.6a and 2.6b)

- $\quad$ Simulate flow through $A V$ valves: If Pressure of atrium > than Pressure of ventricle; $A V$ is fully open $\left(Q=Q_{\max }\right)$; else it is fully closed $(Q=0)$

- Simulate blood pressure and flow through systemic and pulmonary ventricles(Equations 2.3a, 2.3b, 2.4, 2.5a and 2.5b)

- $\quad$ Simulate blood flow through Semi-lunar valves: If Pressure in ventricles $>$ Sinus, Semilunar valve opens fully $\left(Q=Q_{\max }\right)$, else the valve is fully closed $(Q=0)$

- Evaluate and output " $A$ ", " $B$ " or any desired variable

$\circ$ End

\section{* End}

Stop\}

\subsubsection{Simulation of Fixed Points for Obtained Values of A and B Parameters}

The solution to equation 2.13 was sought using the MATLAB imbedded ode23tb solver. This solver implements the trapezoidal rule as a first stage and then the gear formula as the second [35, 36]. Although it is computationally expensive, it gives good accuracy and addresses the issue of instability that may arise peradventure the equation becomes stiff for certain parameter sets [37].

The steady state responses were observed via numerical simulations to determine the system's fixed point, for a range of simulated $A$ and $B$ parameters. The steady state solutions were observed and then the values of angular displacement in radian were plotted against the corresponding parameter to obtain a local bifurcation diagram. 


\subsubsection{Simulation of Phase Portraits for Selected Values of A, B and C}

To verify the stability properties of the equilibrium point, the phase portraits were generated for the different parameters listed below. This was to observe qualitative behaviour for selected parameter sets. The solutions were sought using the MATLAB imbedded ode23tb solver, and then the angular displacements are plotted against corresponding angular velocities to obtain the phase portraits.

1. $\mathrm{C}=-50, \mathrm{~A}=380.5541, \mathrm{~B}=19.3363$ (Negative value of $\mathrm{C}$ )

2. $\mathrm{C}=0, \mathrm{~A}=10.0291, \mathrm{~B}=1$ (When $\mathrm{C}$ is zero - absent of damping or friction)

3. $\mathrm{C}=50, \mathrm{~A}=118771.6265, \mathrm{~B}=1250.4882(\mathrm{C}$-square $>4 \mathrm{D})$

4. $\mathrm{C}=50, \mathrm{~A}=1767.767, \mathrm{~B}=1767.767(\mathrm{C}$-square $=4 \mathrm{D})$

5. $\mathrm{C}=50, \mathrm{~A}=380.5951, \mathrm{~B}=19.3363$ (C-square $<4 \mathrm{D})$

6. $\mathrm{C}=50, \mathrm{~A}=0, \mathrm{~B}=0$ (Pressure and flow equal zero)

7. $\mathrm{C}=50, \mathrm{~A}=15, \mathrm{~B}=10$ (The saddle point)

\section{Results}

\subsection{Simulated Values of Parameters A and B over a Heart Period}

The simulated values of parameter $A$ over a heart period of 1 second for the four valves are shown in Fig. 2. Values of parameter $A$ are higher for the valves in the left heart (Mitral and Aortic Valves) due to higher pressure exerted by the left heart, responsible for pumping blood around the whole body. The curves for the valves on same part of the heart were similar. Simulated values of parameter $B$ over a heart period of 1 second for the four valves are shown in Fig. 3. Values of parameter B were highest in the Aortic valve followed by mitral, pulmonary and tricuspid valves respectively. The curves for the semilunar valves (Aortic and Pulmonary) were similar while those of the AV valves (Mitral and tricuspid) were similar.
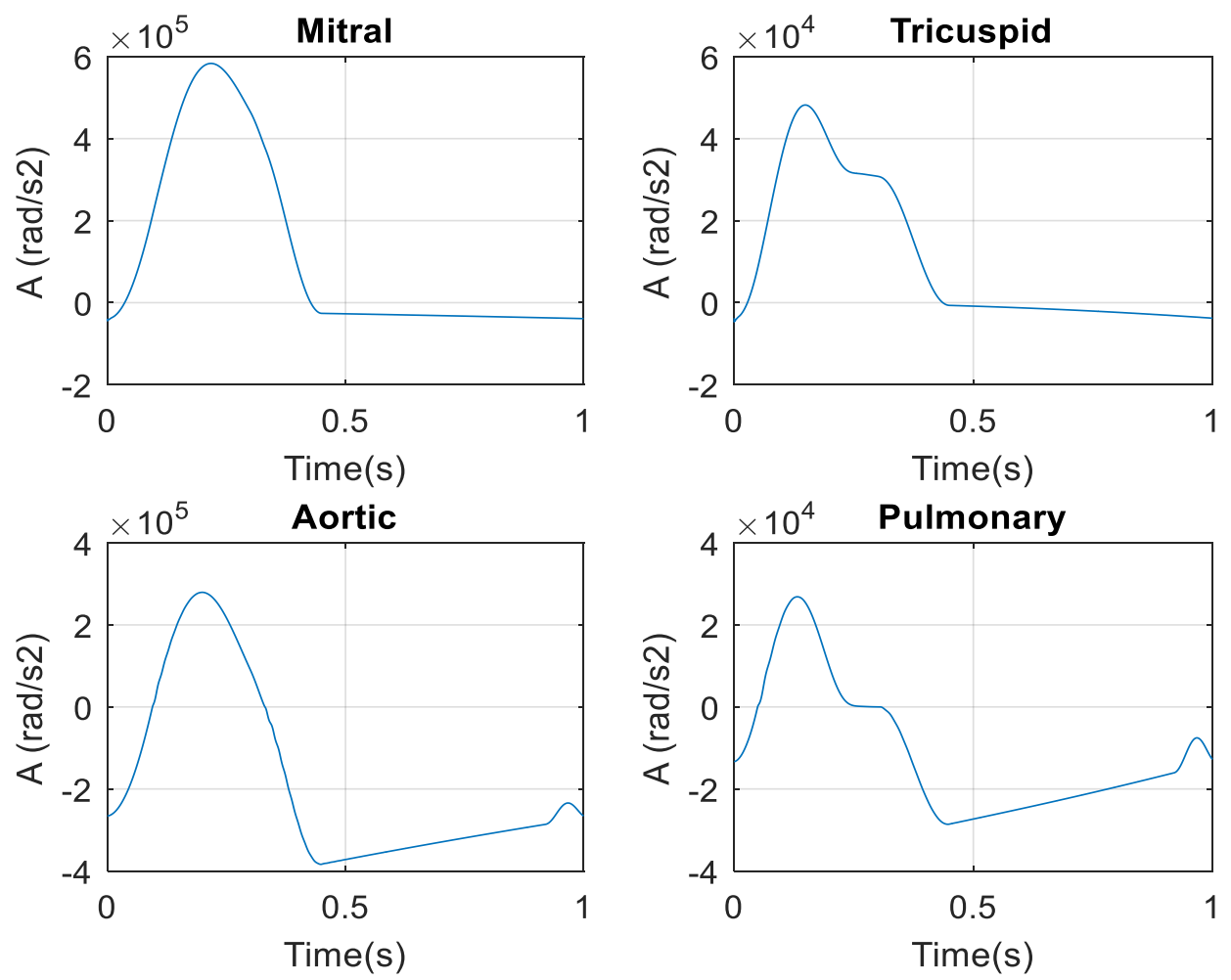

Figure 2. Simulated values of Parameter A over a Heart Cycle. 

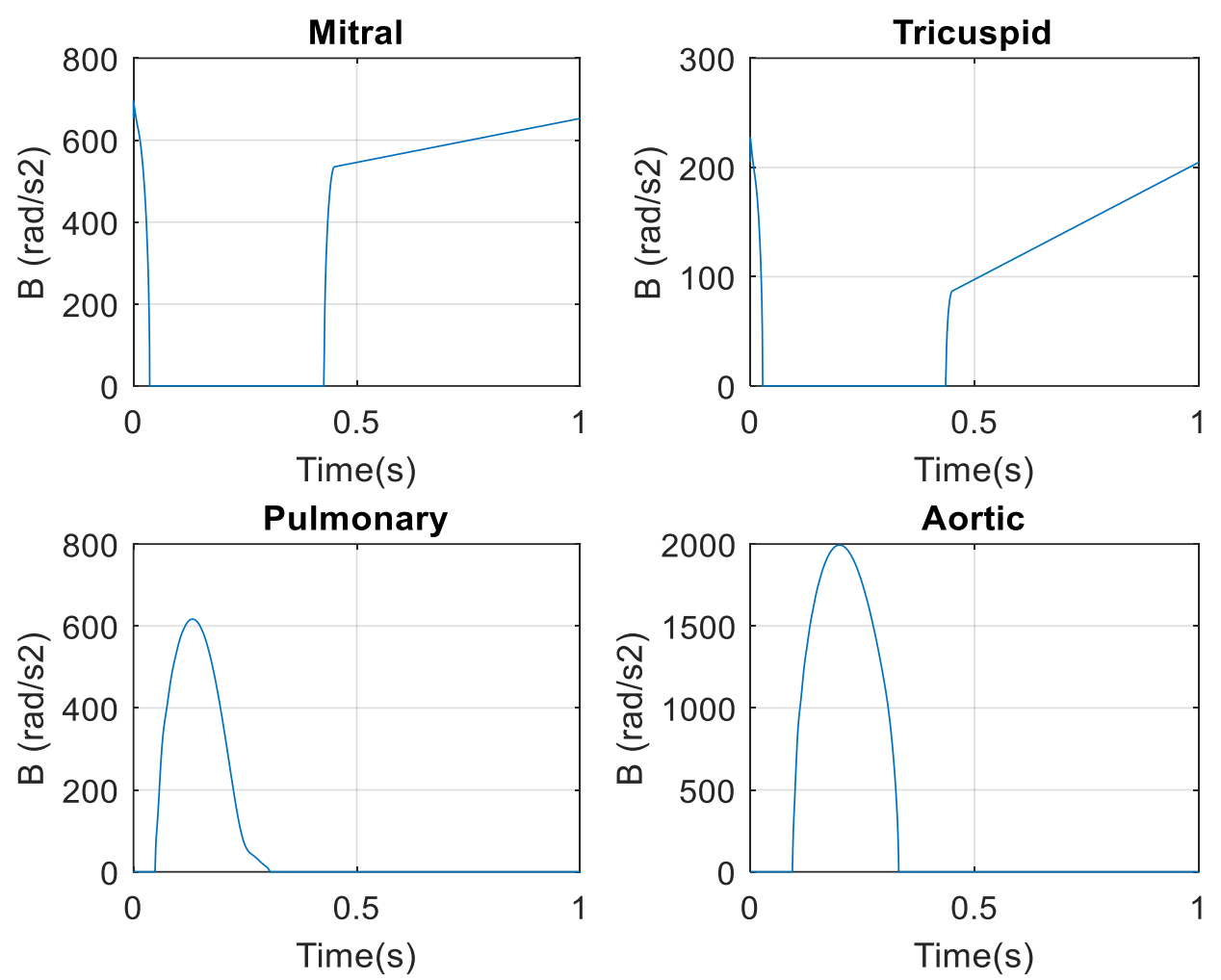

Figure 3. Simulated values of Parameter B over a Heart Cycle

\subsection{Local Bifurcation of Fixed Points}

Fig. 4 is the bifurcation diagram showing the two fixed points for the simulated range of $A$ and $B$ parameters for the aortic valve. Similar bifurcation diagram were observed for the other valves. The blue dots are the stable node which the system finally settles to. While the red dots are saddle points (unstable nodes). The stable node is the observed steady angular displacement when equation 2.13 was solved with ode23tb for the simulated A and B parameters. The unstable nodes / saddle points (red dots) were evaluated by adding $\pi$ and then expressed in polar form (between 0 and $2 \pi$ ). As observed, there is exchange of stability between the fixed points when $A$ or $B$ equal to zero. The value of stable fixed point (blue dots) for a positive values of $A$ and $B$ lies in the first quadrant (between zero and $\pi / 2$ radians) while the unstable fixed points (red dots) lies in the third quadrant (between $3 \pi / 4$ and $2 \pi$ ). For negative values of $A$, the first fixed point $\left(\theta_{1}^{*}, 0\right)$ becomes unstable while the second fixed point $\left(\pi+\theta_{1}^{*}, 0\right)$ becomes stable. 


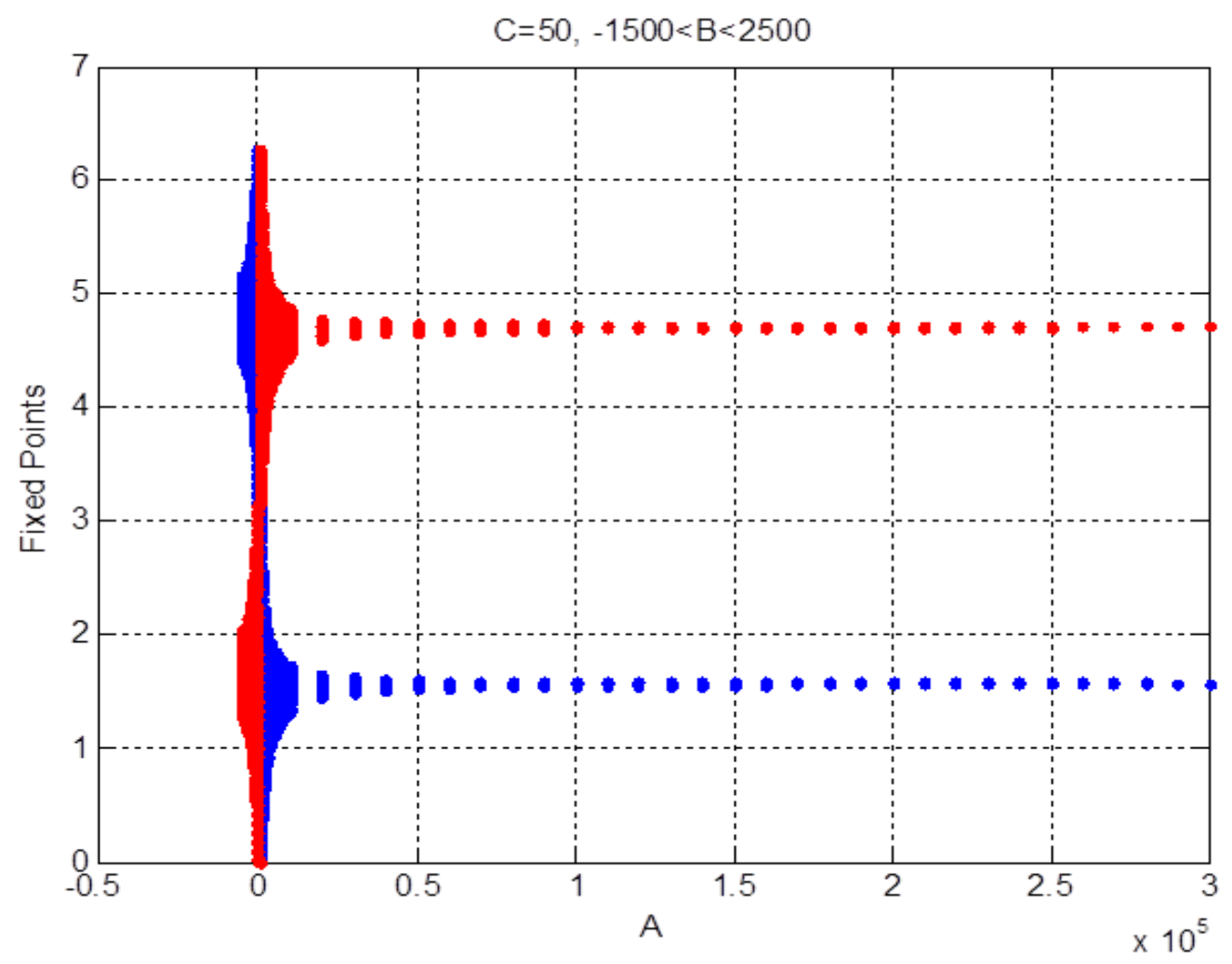

Figure 4. Local bifurcation plot as parameters $A$ and B were varied

\subsection{Linear Stability Analysis}

Equations $2.23 \mathrm{a}$ and $2.23 \mathrm{~b}$ are the evaluated eigenvalues of the system's Jacobian matrix. The dynamics in a finite region around the fixed point is determined by their stability properties which are explored as follows.

Considering the simple scheme for classifying fixed points [32,34]; the stability of the system is a function of parameter $C$. Fig. 5 shows increasing values of angular displacements and velocity as time progresses indicating that the system is not stable. A positive coefficient of $C$ in Equation 2.13 will result in a positive real part of the eigenvalues which is the characteristic of an unstable system. When $C$ is equal to zero, the system is marginally stable, hence as observed in Fig. 6, the system settles into a circle that passes through its initial conditions in the state space.

The disappearance of fixed points for values of $C$ less than zero $(0)$ describes a saddle-node bifurcation in the model. The motion of the system when $C=0$ can be described as a circle. In such case, the system is semi-stable and rotates through the initial conditions forming a circle. Positive values of $C$ will yield a stable motion. 


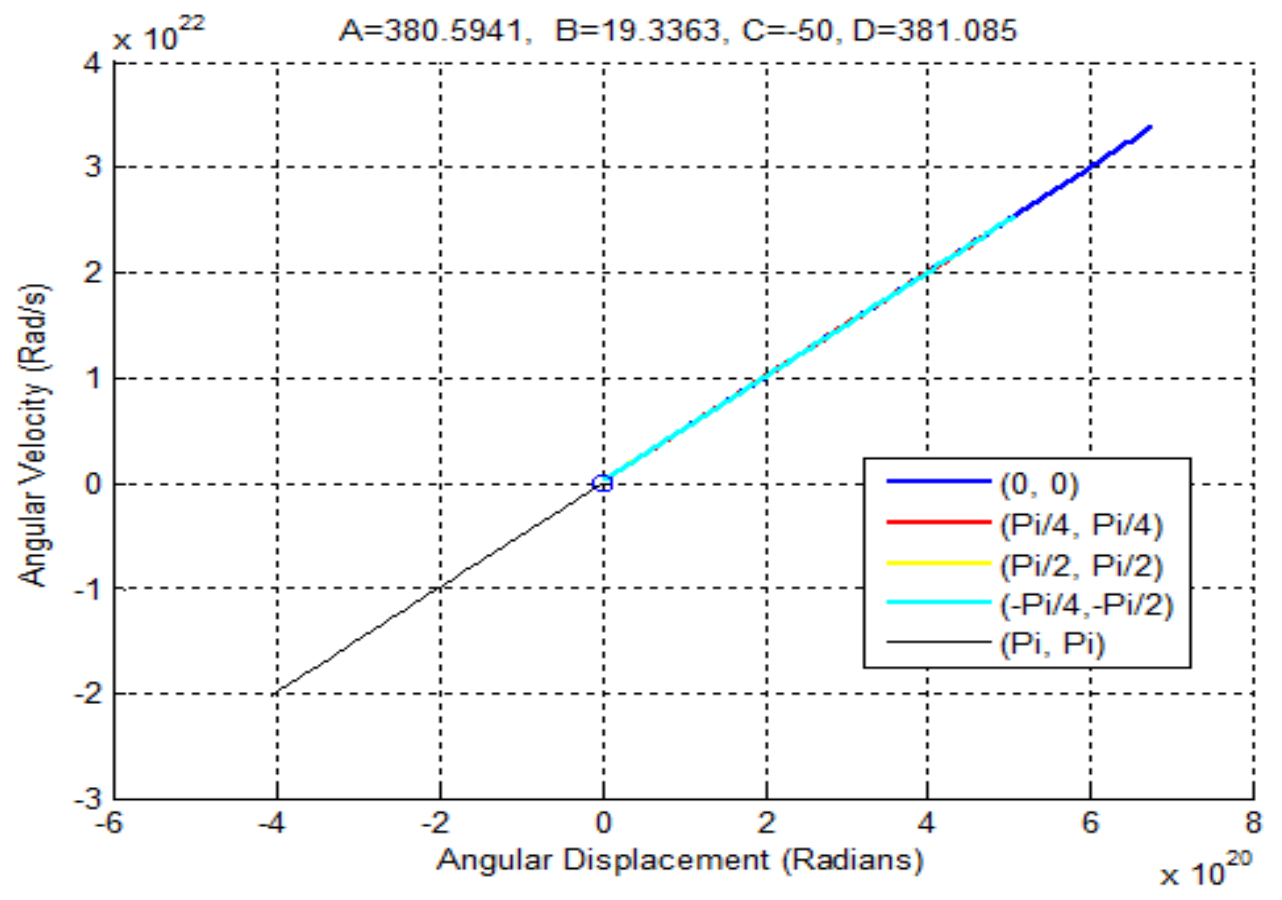

Figure 5. Phase plot for a negative value of parameter $C$. Showing the solution tending to infinity for both velocity and displacement

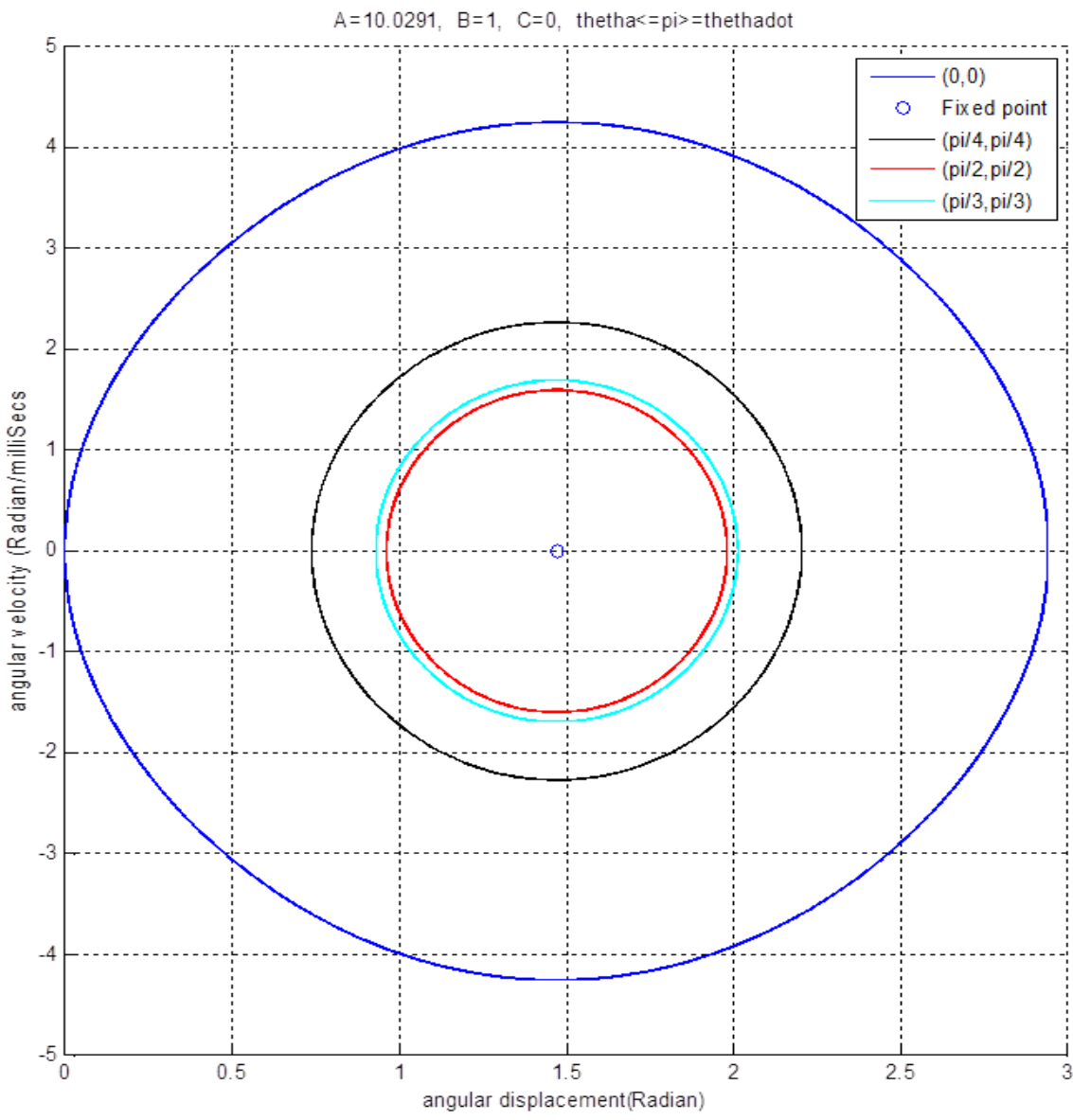

Figure 6. Phase plot of the steady state solution obtained for different initial conditions between 0 and $\pi$ radians

A negative coefficient of $C$ in Equation 2.13 will give a stable equilibrium solution for one of the fixed point and a saddle for the second fixed point. The nature of a saddle point can be envisioned in Fig. 11. 
The stability property of the stable fixed point is a function of $D$ (Equation 2.21). When the value of $C^{2}$ is lesser than $4 D$, the first fixed point $\left(\theta_{1}^{*}, 0\right)$ is a stable spiral having a root which is a complex conjugate pair. Hence any initial condition around the fixed point will spiral into the fixed point as observed in Fig. 7. The second fixed point $\left(\pi+\theta_{1}^{*}, 0\right)$ is a saddle having two eigenvalues which are real but of opposite sign. As the value of $D$ increases, the number of spirals reduces. As shown in Fig. 8, the phase trajectory spirals just ones settling to the fixed point.

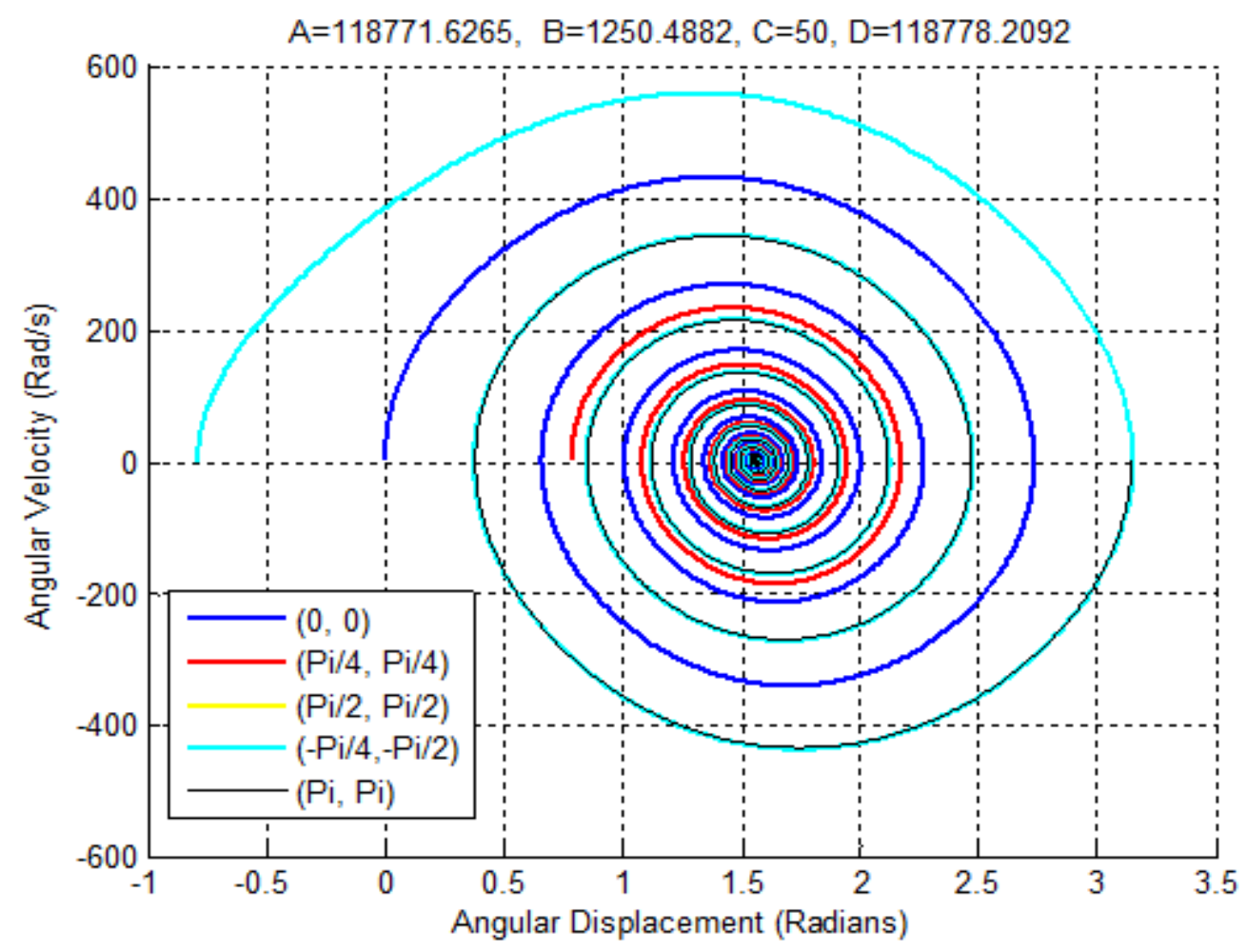

Figure 7. Phase plot of the steady state solution obtained for different initial conditions for $C$ Square lesser than 4D

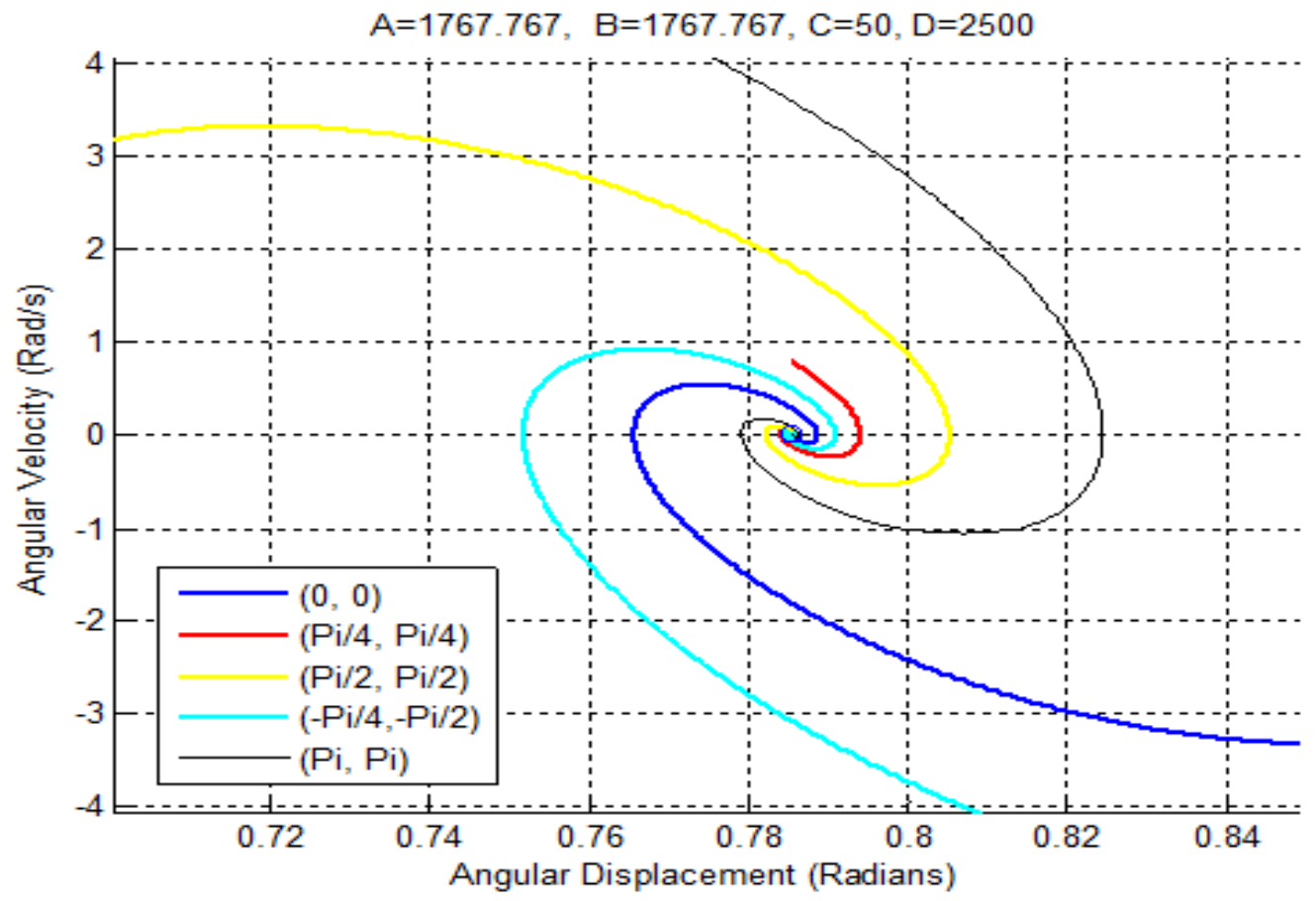

Figure 8. Phase plot of the steady state solution obtained for different initial conditions for $C$ square equal to $D$ 
When the value of $C^{2}$ is equal to or less than $4 D$, the first fixed point $\left(\theta_{1}^{*}, 0\right)$ is a stable degenerative node having two real and equal (same sign) eigenvalues. Hence any initial condition around the fixed point will settle into the fixed point as observed in Fig. 9. The second fixed point $\left(\pi+\theta_{1}^{*}, 0\right)$ is a saddle having two eigenvalues which are real but of opposite sign.

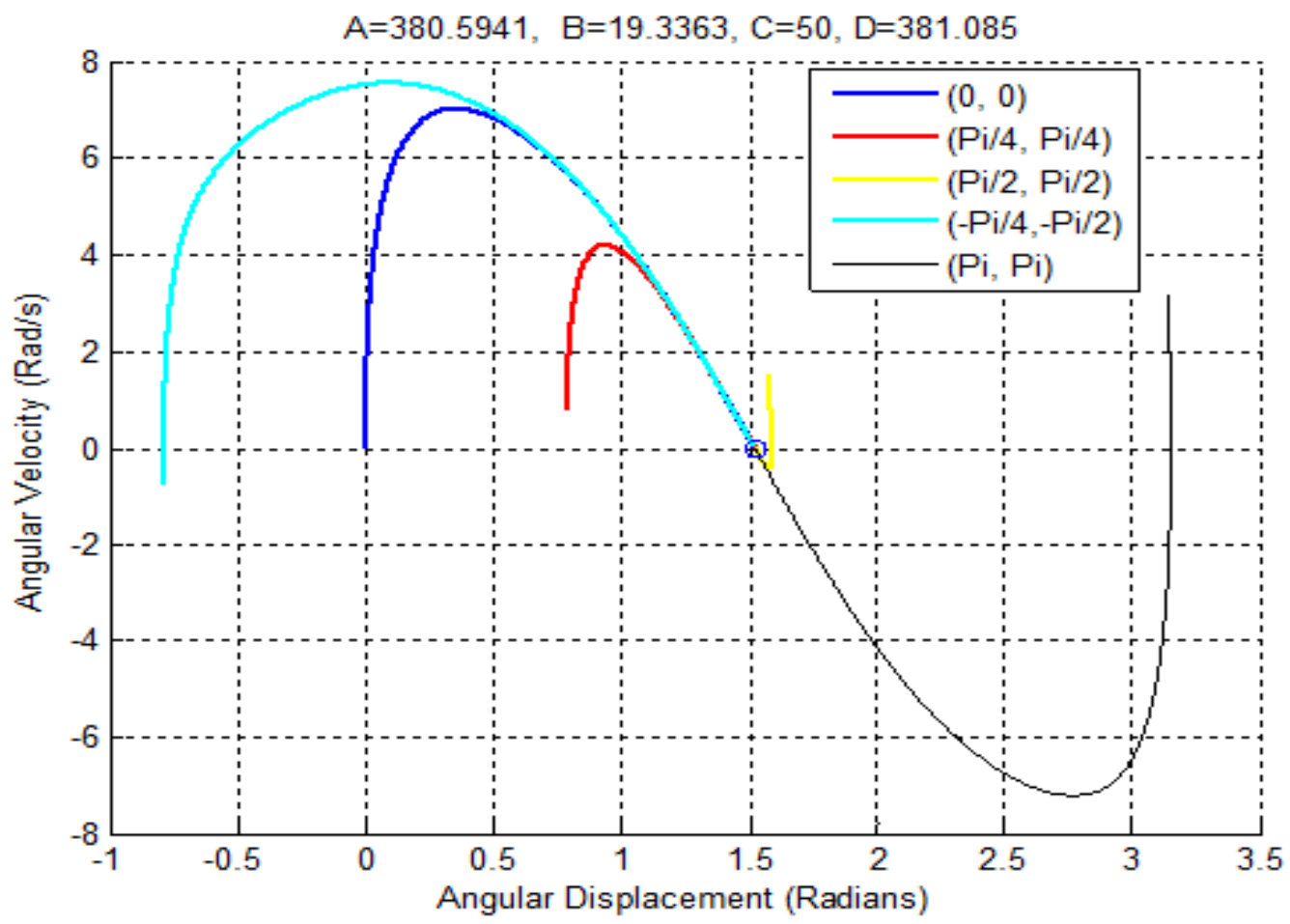

Figure 9. Phase plot of the steady state solution obtained for different initial conditions for Csquare greater than $\mathrm{D}$

Fig. 10 shows a number of vertical lines for $\mathrm{D}$ is equal to zero $(\mathrm{A}=0, \mathrm{~B}=0)$. It is an indication that the system rapidly comes to rest without any displacement.

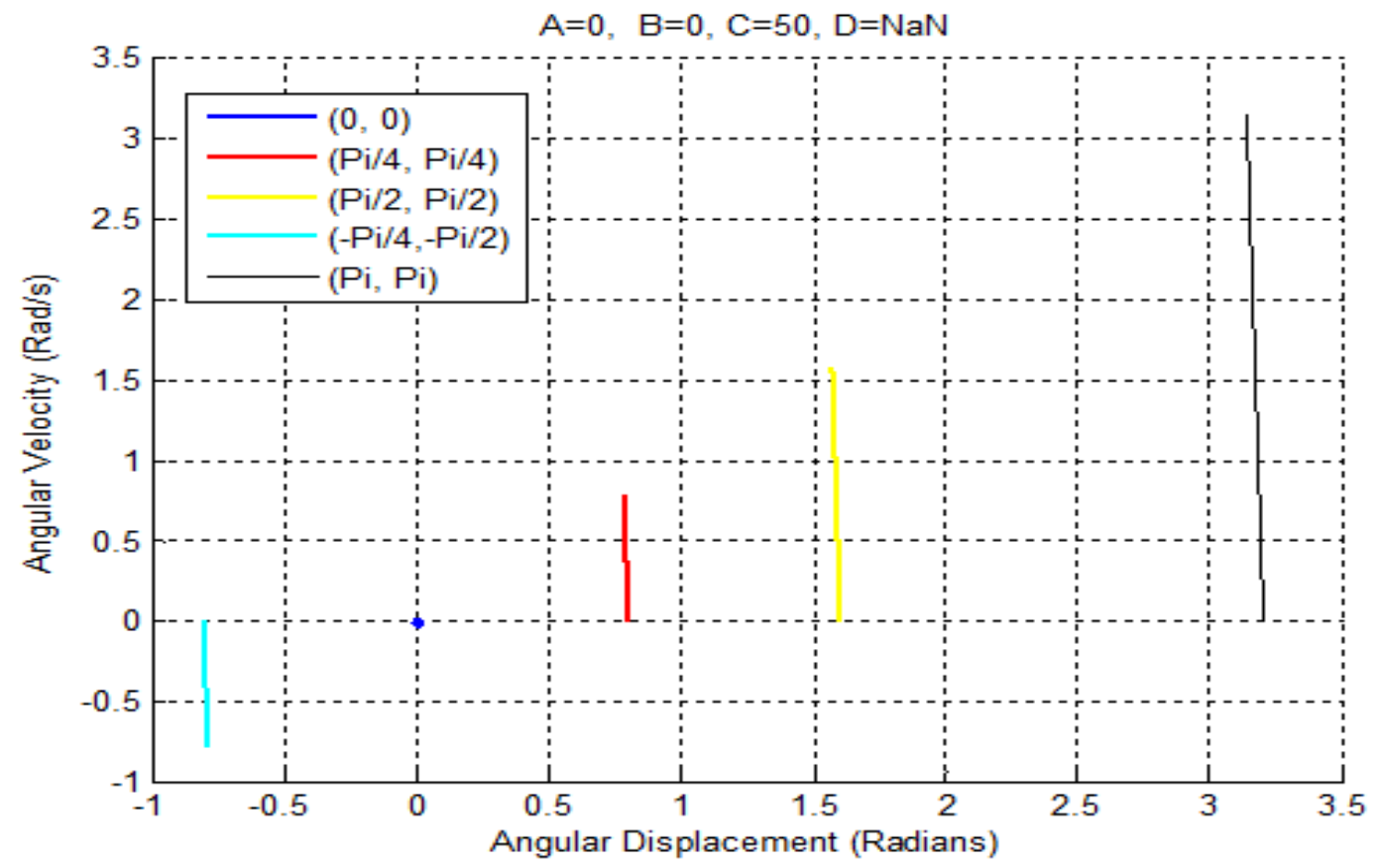

Figure 10. Phase plot of the steady state solution obtained for different initial conditions for $\mathrm{A}=0, \mathrm{~B}=0$ 
Fig. 11 shows different equilibrium positions (fixed points) for different initial conditions when 4D is far less than $C^{2}$.

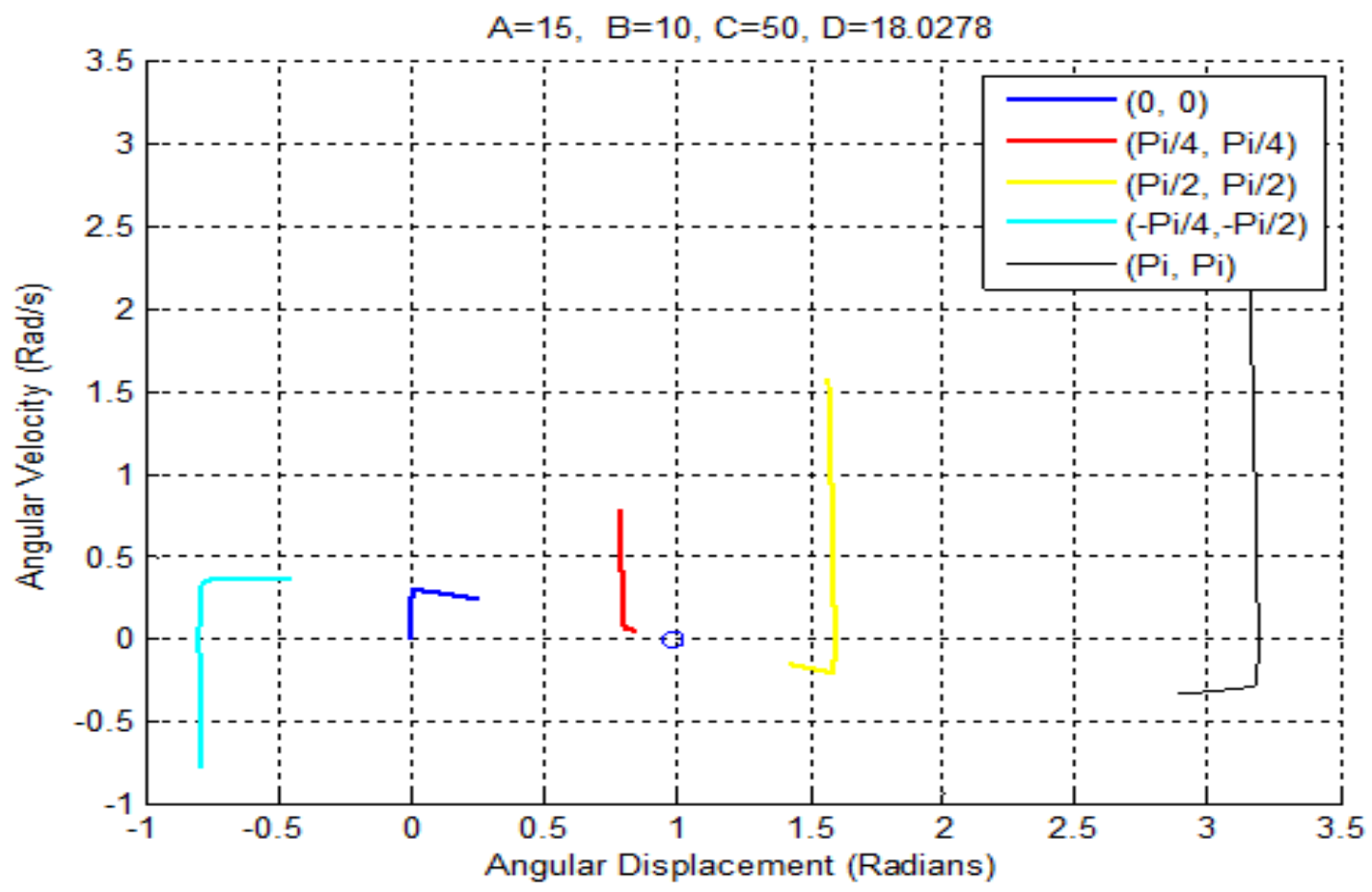

Figure 11. Phase plot of the steady state solution obtained for different initial conditions for $A=15, B=10$

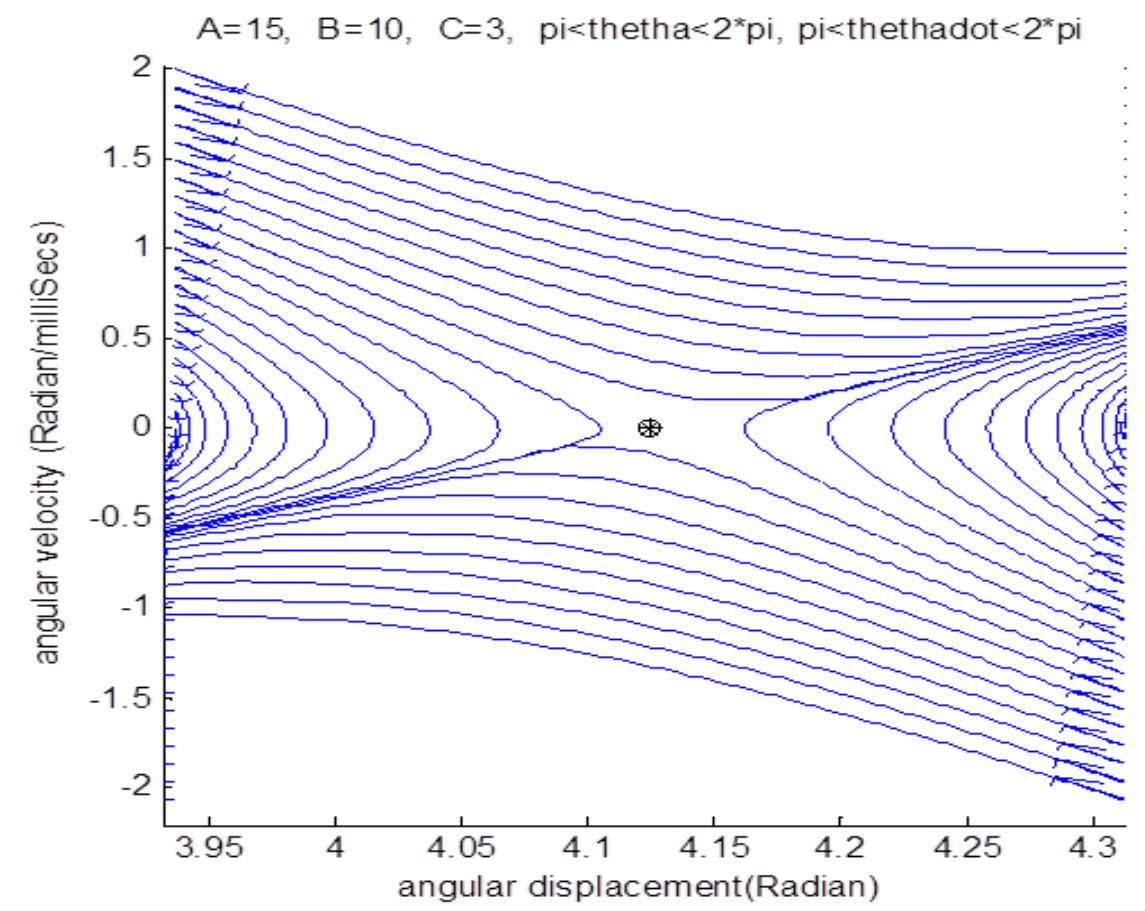

Figure 12. Vector field around a pre-determined saddle node fixed point for parameter $C$-square lesser than $D$

\section{Discussions and Concluding Remarks}

The motion of heart valve leaflet is characterised by two fixed points which is always between $-2 \pi$ and $2 \pi$. One is usually a stable node and the other a saddle point. The leaflet will always move towards the stable fixed point. The fixed points depends solely on blood pressure and flow effect and 
is unaffected by the damping properties of the heart valve leaflet. The value of the stable fixed point is a function of the ratio $A$ (pressure and flow effect) to $B$ (vortices or any effect underneath the leaflet). As the ratio tends towards infinity, the fixed point tends toward $\pi / 2$ radians (90 degrees). Other effects such as clothed blood beneath the leaflet may increase the value of $B$, thereby decreasing the ratio of $A$ to $B$ and can possibly cause reduced leaflet motion.

The sense of the ratio $A: B$ determines which of the fixed points is stable. Different instances of pressure differential across valve and direction of flow are discussed in the literature [29, 38]. For example, the systolic opening of the semilunar valves in which higher ventricular pressure and forward blood flow keeps the valve open is a situation of positive pressure gradient and flow in the positive direction, which will yield a positive ratio. Rapid blood flow observed in semilunar valves for few milliseconds at the beginning of diastole is an example of a negative pressure difference across the valve and a positive blood flow, yielding a negative ratio.

Considering that the value of $C$ (tissue friction or damping effect) is constant and always positive, the motion of the heart valve leaflet is always stable due to the effect of friction. However, their stability properties may change as parameters $A$ and $B$ are changing within a heart cycle. The direction of the valve leaflet is strongly influenced by the pressure difference across the valve and blood flow. Stability analysis of the fixed points shows that the stable fixed point is always a spiral, star node or degenerative node while the other is always a saddle node. Since all saddle nodes are usually unstable [32], the direction of the valve leaflet will always be towards the stable fixed point irrespective of its position in the phase plot. The stability property of the fixed point is a function of all the factors affecting the valve motion. When the value of $C^{2}$ is less than $4 D$ the motion is a spiral while a stable node when $D$ is greater than $D$ indicates rapid convergence towards the fixed points due to rapid decay. This explains the slow valve opening at onset and rapid valve closure observed by Leyh et al. [39].

It can be assumed that only the stable fixed point occurs since all trajectories will eventually settle to it. The stable fixed point is equivalent to "ARCTAN the ratio of $A: B$ ". There are different possible scenarios of heart valve motion within a heart cycle. Zero value of fixed point results when the flow across the valve and pressure gradient are both zero such as in the onset of diastolic state in a heart chamber. This situation may occur over an infinitesimal time as the flow and pressure parameters are changing continuously over a heart cycle. Negative values of fixed points for angular displacement observed for negative values of ratio A to B is an indication of the valve leaflet opening inwards, a medical condition called prolapse. The tendon chords in AV valves support the valve and provides mechanical load during systole and helps prevent prolapse. For the semilunar valves, prolapse is prevented by the annulus and the moonlike shape formed when the valve closes [38]. A model of this mechanical force that prevents prolapse in the AV valve can be seen in the work of Toma et al. [40].

\section{Conclusion}

In this paper, we studied the kinetics of the human heart valve leaflet, by investigating its fixed points and stability properties with the KS heart valve model. From our investigation, the motion of the heart valve is always stable due to the effect of friction. The direction of the valve leaflet is strongly influenced by the pressure difference across the valve and blood flow. The switching of stability of the fixed points indicates that the motion of valve leaflet undergoes a transcritical bifurcation within a heart cycle. The KS model was successfully used to assess how the motion of the valve evolves in state space as the parameters changes. 


\begin{tabular}{|llll|}
\hline Nomenclature & Subscript & \\
\hline $\mathrm{I}$ & Second moment of Inertia & $\mathrm{a}$ & atrium \\
$\theta$ & Angular displacement & $\mathrm{V}$ & ventricle \\
$\theta^{\prime}$ & Angular velocity & $\mathrm{min}$ & minimum value \\
$\theta^{\prime \prime}$ & Angular acceleration & $\mathrm{max}$ & maximum value \\
$\mathrm{V}$ & Volume & $\mathrm{in}$ & inlet value \\
$\mathrm{P}$ & Pressure & $\mathrm{out}$ & outlet value \\
$\mathrm{t}$ & Time & $\mathrm{d}$ & diastolic \\
$\mathrm{E}$ & Elastance & $\mathrm{S}$ & systolic \\
$\mathrm{e}$ & Activation function & $\mathrm{T}$ & time \\
& & & Peak of systolic \\
$\mathrm{T}$ & Heart period & $\mathrm{s} 1$ & phase \\
& & & End of systolic \\
$\mathrm{C}$ & Vascular capacitance & $\mathrm{s} 2$ & phase \\
& & & Beginning of $\mathrm{P}$ \\
$\mathrm{R}$ & Vascular resistance & $\mathrm{Pb}$ & wave \\
$\mathrm{L}$ & Vascular inductance & $\mathrm{Pw}$ & Duration of $\mathrm{P}$ wave \\
$\mathrm{A}$ & First valve lump parameter & $\mathrm{As}$ & aortic sinus \\
$\mathrm{B}$ & Second valve lump parameter & $\mathrm{Vn}$ & vein \\
$\mathrm{C}$ & Third valve lump parameter & $\mathrm{At}$ & artery \\
$\mathrm{D}$ & Sum of A square and B square & $\mathrm{Cp}$ & capillary \\
$\mathrm{K}$ & Coefficient & $\mathrm{Av}$ & aortic valve \\
\hline
\end{tabular}

\section{Conflict of Interest}

The authors declare that they have no conflict of interest.

\section{Acknowledgments}

The authors wish to acknowledge the Tertiary Education Trust Fund (TETFund) for the studentship they provided to Emagbetere Eyere, while undertaking his Ph.D. program.

\section{References}

[1] K. Huynh, Thrombosis leaflet motion after TAVI or SAVR, Nature Reviews Cardiology. 12 (2015).

[2] R.R. Makkar et al., Possible subclinical leaflet thrombosis in bioprosthetic aortic valves, The New England Journal of medicine. 373(21) (2015) 2015-2024.

[3] J. C. Laschinger et al., Reduced leaflet motion in bioprosthetic aortic valves - the FDA perspective, The New England Journal of Medicine. 373(21) (2015) 1996-1998.

[4] D. Magnus et al., Hypo-attenuated leaflet thickening and reduced leaflet motion in sutureless bioprosthtic aortic valves, J. Am. Heart Assoc. 2017(6) (2017).

[5] W.A. Seed, N.B. Wood, Development and evaluation of hot-film velocity probe for cardiovascular studies, Cardiovasc. Res. 4 (1970) 253-263.

[6] H.L. Falsetti et al., Sequential velocity development in the ascending and descending aorta of dog, Circulation Research 31(3) (1972) 328-338.

[7] M.J. Thubrikar, The aortic valve, CRC Press, Borca Raton, Florida, 1990.

[8] Z. He et al., In vitro dynamics strain behaviour of the mitral valve posterior leaflet, Transcactions of ASME. 127 (2005) 504-511. 
[9] J.T. Fenner, W.M. Mackay, D. Wheatney, Laser profiling: a technique for the study of prosthetic leaflet heart valve motion, Physiol. Meas. 16 (1995).

[10] A.K. Iyengar et al., Dynamics In Vitro Quantification of Bioprosthetic Heart Valve Leaflet Motion Using Structured Light Projection, Annals of Biomedical Engineering. 29 (2001) 963973.

[11] A.G.B. Donn et al., Laser profiling of bovine pericardial heart valves, Artif. Organs. 20 (1997) 436-439.

[12] G. Pache et al., Early hypo-attenuated leaflet thickening in balloon-expandable transcatheter aortic heart valves, European Heart Journal. 37 (2016) 2263-2271.

[13] R. Yanagisawa et al., Incidence, predictors, and mid-term outcomes of possible leaflet thrombosis after TAVR, JACC: Cardiovasc. Imaging. 10(1) (2016) 1-11.

[14] E. J.Weinberg, D. Mofraad, M.R. Shahmizradi, On the multiscale modelling of heart valve biomechanics in health and disease, Biomech. Model Mechanobiol. 9 (2010) 373-387.

[15] R. Zakerzadeh, M.-C. Hsu, M.S. Sacks, Computational methods for the aortic heart valve and its replacements, Expert Review of Medical Devices. 14(11) (2017) 849-866.

[16] I. Borazjani, A review of fluid-structure interaction simulations of prosthetic heart valves, Journal of Long-Term Effects of Medical Implants. 25 (2015) 75-93.

[17] A. Joda et al., Multiphysics simulation of the effect of leaflet thickness inhomogeneity and material anisotropy on the stress-strain distribution on the aortic valve, Journal of Biomechnics. 49(12) (2016) 2502-2512.

[18] H. Gao et al., A coupled mitral valve-left ventricle model with fluid-structure interaction. Journal of Medical Engineering \& Physics. 47 (2017) 128-136.

[19] R. Mittal et al., Computational modeling of cardiac hemodynamics: current status and future outlook, Journal of Computational Physics. 305 (2016) 1065-1082.

[20] B. Su et al., Cardiac MRI based numerical modeling of left ventricular fluid dynamics with mitral valve incorporated, Journal of Biomechanics. 49(7) (2016) 1199-1205.

[21] Z.C. Wang et al., Computational modeling for fluid-structure interaction of bioprosthetic heart valve with different suture density: comparison with dynamic structure simulation, International Journal of Pattern Recognition and Artificial Intelligence. 31(11) (2017).

[22] G. Luraghi et al., Evaluation of an aortic valve prosthesis: Fluid-structure interaction or structural simulation?, Journal of Biomechanics. 58 (2017) 45-51.

[23] W. Mao et al., Fully-coupled fluid-structure interaction simulation of the aortic and mitral valves in a realistic 3D left ventricle mode, PLOS ONE. 12(9) (2017).

[24] Y. Shi, P. Lawford, H. Rodney, Review of Zero-D and 2-D models of blood flow in the Cardiovascular system, PLOS ONE. (2011).

[25] J. Warner, D. Bohringer, M. Hexamer, Simulation and prediction of cardiotherapeutical phenomena from a pulsatile model coupled to the guyton circulatory model, IEEE Transactiosn on Biomedical Engineering. 49(5) (2002) 430-439.

[26] Y. Shi, T.J.H. Yeo, Y. Zhao, Numerical simulation of a systemic flow test rig, ASAIO Journal. 50(1) (2004) 54-64.

[27] A. Laadhari, A. Quarteroni, Numerical modeling of heart valves using resistive Eulerian surfaces, International Journal for Numerical Methods in Biomedical Engineering. 32(5) (2016) e02743. 
[28] T. Korakianitis, Y. Shi, Numerical simulation of cardiovascular dynamics with healthy and diseased heart valves, Journal of Biomechanics. 39 (2006) 1964-1982.

[29] A.C. Guyton, J.E. Hall, Textbook of Medical Physiology, Elsevier Inc., Philadelphia, Pennsylvania, 2006.

[30] R.M. Berne, Cardiovascular Physiology, The C.V. Mosby Company, St. Louis, MO. 1981.

[31] E. Kreyszig, Advance Engineering Mathematcs, $9^{\text {th }}$ edition, John Wiley \& Sons, INC., Singapore, 2006.

[32] S.H. Strogatz, Non-linear Dynamics and Chaos with Applications to Physics, Biology, Chemistry, and Engineering. Reading, Massachusetts: Perseus Books Publishing, 1994.

[33] J.R. Taylor, Classical Mechanics, University Success Books, 2005.

[34] F.C. Moon, Chaotic Vibrations: An Introduction for Applied Scientist and Engineers. John Wiley and Sons Inc, New Jersey, 2004.

[35] M. Natick, Using MATLAB version 5.1, The Matworks, 1997.

[36] A. Ryuichi, N. Michihiro, V. Remi, Behind and Beyond the MATLAB ODE suite. CRM-2651, 2000 .

[37] E. Emagbetere, O. Oluwole, T.A. O. Salau, Comparative study of Matlab ODE solvers for the Korakianitis and Shi model, Bulletin of Mathematical Sciences and Applications. 19 (2017) 3144.

[38] J.D. Bronzino, The Biomedical Engineering handbook, CRC Presss, Boca Raton, 1995.

[39] R.G. Leyh et al., Opening and closing characteristics of the aortic valve after different types of valve-preserving surgery, Circulation. 100(21) (1999) 2153-2160.

[40] M. Toma et al., Fluid-structure interaction analysis of ruptured mitral chordae tendinae, Annals of Biomedical Engineering. 45(3) (2017) 619-631. 